This item was submitted to Loughborough's Research Repository by the author.

Items in Figshare are protected by copyright, with all rights reserved, unless otherwise indicated.

\title{
Development of speckle shearing interferometer error analysis as an aperture function of wavefront divergence
}

PLEASE CITE THE PUBLISHED VERSION

PUBLISHER

(c) Taylor \& Francis

LICENCE

CC BY-NC-ND 4.0

\section{REPOSITORY RECORD}

Abdullah, Wan S. Wan, and Jon N. Petzing. 2019. "Development of Speckle Shearing Interferometer Error Analysis as an Aperture Function of Wavefront Divergence". figshare. https://hdl.handle.net/2134/3637. 
This item was submitted to Loughborough's Institutional Repository by the author and is made available under the following Creative Commons Licence conditions.

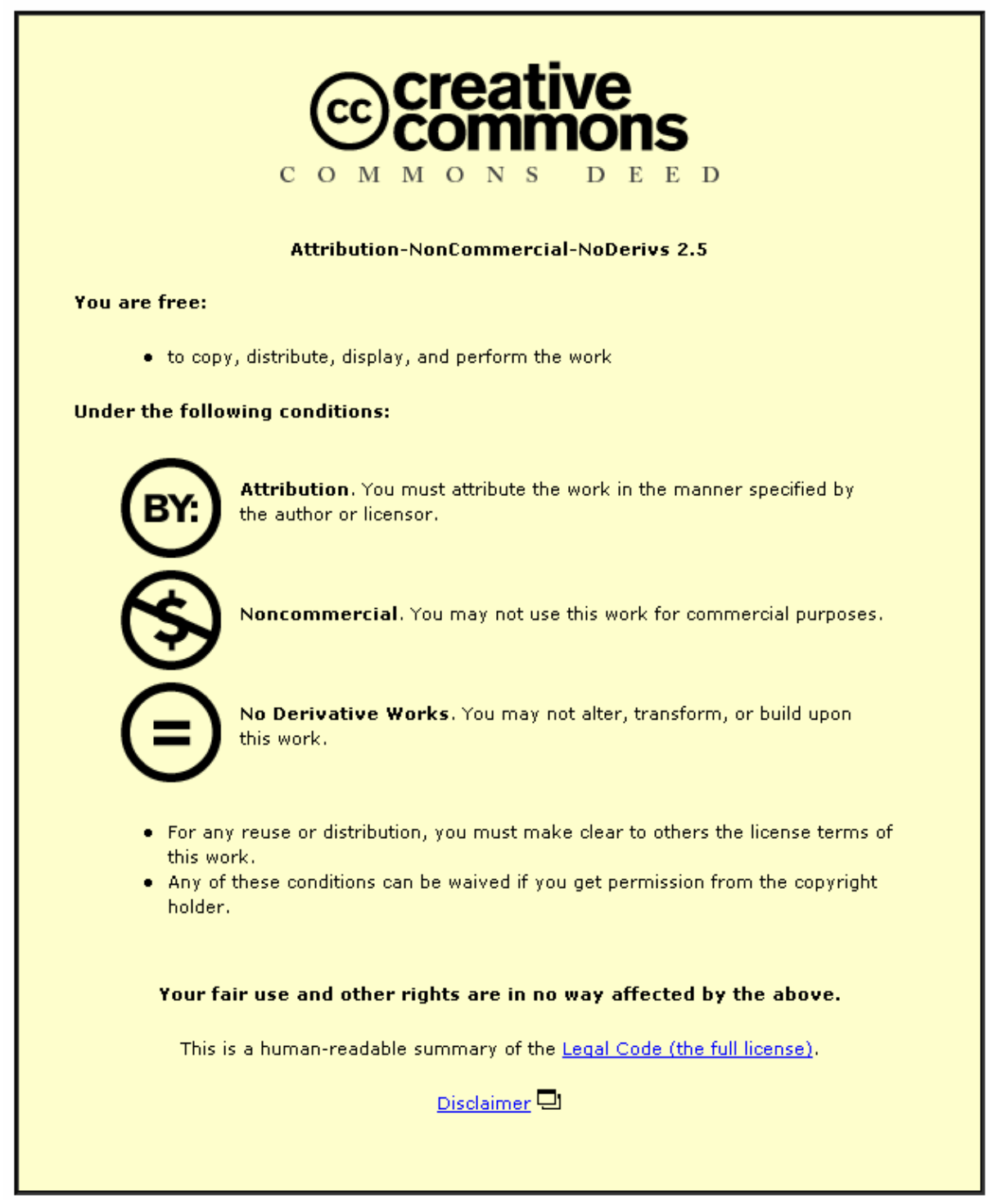

For the full text of this licence, please go to: http://creativecommons.org/licenses/by-nc-nd/2.5/ 

Malaysia

Contact Details:

Dr Jon Petzing

Leicestershire

Tel: 01509227617

Fax: 01509227648

E-mail:j.petzing@lboro.ac.uk 


\section{Abstract}

28 Increasing the confidence in wholefield speckle based optical metrology transducers

29 requires a detailed understanding of the error sources of the respective instruments.

30 The analysis of error contributions to the optical phase output of a Michelson based 31 speckle shearing interferometer have been modelled. Specific attention has been made

32 to the effect of the aperture at the image plane, with respect to collimated and non-

33 collimated object illumination. This modelling presents an advance on a previous 34 modelled analytical relationship, which includes partial displacement derivative terms 35 and components as a function of illumination geometries and importantly aperture 36 effects. The work has identified a phase error contribution due to the aperture function 37 of between $0.15 \%$ and $1.48 \%$, dependant on the object distance, when considering a 38 planar object undergoing predominantly surface to normal deformation.

40 Keywords: speckle, shearing, shearography, errors, repeatability 
43 The development of the speckle shearing interferometer [1] has been characterised by

44 several common technological phases, which can be summarised as; invention,

45 demonstration, application. The technique can be based on a number of differing

46 optical designs, but all have a common ability of at least measuring the out-of-plane

47 (normal to the object surface) first order partial displacement derivatives $(\partial \mathrm{w} / \partial \mathrm{x}$ and

$48 \quad \partial \mathrm{w} / \partial \mathrm{y})$. In certain cases, the optical design can be manipulated such that in-plane

49 displacement derivatives such as $\partial \mathrm{u} / \partial \mathrm{x}$, and even second order partial derivatives

$50 \quad\left(\partial^{2} \mathrm{w} / \partial \mathrm{x}^{2}\right)$ can be measured (although not generally in a real-time discreet manner).

52 The development of the speckle shearing interferometer in recent years has been aided

53 by the dramatic changes in laser technology, image processing hardware and software.

54 This has led to increased commercialisation of instrumentation and rising interest from

55 various industrial sectors, which view the instrument data as being suitable for

56 quantified defect and even possibly elements of strain analysis.

Generating quantified data from such a transducer or measurement instrument is but one step towards eventually solving an engineering problem, because the user must have confidence in the measurement transducer. This confidence is generally gained by understanding and quantifying all of the sources of error and uncertainty associated with

62 the instrument, and then applying a calibration strategy which identifies the levels of

63 error and accuracy for the instrument, which allows the linking of the measurand to the

64 primary standard (the metre in this case). In certain circumstances where an instrument 
error analysis is not available, this measurement confidence may be gained from statements of instrument repeatability [2]

Significant work has been completed generally within speckle metrology to understand various issues of error and uncertainty, with several very recent publications now pushing the issues of instrumentation quality and data confidence $[3,4]$. Until recently, when considering speckle shearing interferometry specifically, analysis had typically been limited to issues concerning fringe visibility [5-8], and cross-sensitivity between displacement and displacement derivative components $[9,10]$. More recent published works have started to investigate other error sources, including the lateral shearing amount, displacement derivative order, sensitivity vector, rigid body motion and geometry effects $[11,12]$.

Another issue, which has also been reported, is that of quantified errors being generated as a function of non-collimated illumination. A ray based theoretical and experimental analysis of a Michelson based speckle shearing interferometer [13,14] suggested that significant errors (up to $10 \%$ ) could be inadvertently generated due to object illumination wavefront geometries. Vector based analysis supports these findings and has furthered aspects of this work [15]. Further work has now been completed in this area, which has enhanced the original theoretical model [13], and helps to refine the mathematical model of a simplified case for a speckle shearing interferometer. It is the 


\subsection{Analysis of the theoretical system}

90 Much of the initial development of speckle shearing interferometers for optical 91 metrology applications, can originally be traced to a small number of key publications

92 by a number of researchers [16,17]. Significant work [18,19] subsequently

93 demonstrated the use of wedge based optics for the application of speckle shearing 94 interferometers to deformation analysis. One of the core features of this work (and 95 further work by other authors using alternative optical configurations including the 96 Michelson based optics) is the mathematical description used for the analysis of the 97 optical phase signals $(\Delta)$ produced by the interferometer, which was identified as being 98 applicable to Michelson or wedge based speckle shearing interferometers:

$$
\Delta=\frac{2 \pi}{\lambda}\left[(1+\cos \theta) \frac{\partial w}{\partial x}+\frac{\partial u}{\partial x} \sin \theta\right] \delta x
$$

where ' $\lambda$ ' is the laser wavelength, ' $\theta$ ' is the angle of illumination, $\partial w / \partial x$ and $\partial u / \partial x$ represent out-of-plane and in-plane first order displacement derivatives, and $\delta x$ is the

This one relationship forms the basis for many subsequent works and texts by many other authors, providing a first order approximation to the true optical phase value. The issue of suitability of equation 1 has previously been discussed by the current authors $[13,14]$, where it is correctly identified that this is a first order approximation, because the theoretical formulation involves a Taylor series [18,19], which includes second, third and higher order derivatives. However, it has been shown that typically, these 
112 higher order derivatives may only become significant if a large lateral shear is used $(\delta x)$,

113 and even then the second order partial derivative will only contribute a few percent to

114 the overall numerical analysis of the fringe function.

115

116 An important issue which is not often highlighted in research or trade publications, is 117 that equation 1 is based on an analysis of one point at the object plane, and is 118 consequently only truly valid for that one point at the surface normal, and importantly, 119 when using collimated object illumination. However, this relationship is commonly 120 used for the general wavefront approximations, and calculation of optical deformation 121 phase across the entire illumination wavefront.

123 Existing work [13,14], which has analysed the consequence of using non-collimated 124 illumination for a speckle pattern interferometer, resulted in a theoretical extension to 125 equation 1 which was experimentally verified and correlated. This new formulation 126 (equation 2) suggested that two additional error terms (equation 3) should be included 127 within the phase description, which would compensate for the use of non-collimated 128 object illumination, on a planar object.

129

130

$$
\Delta_{x}=\frac{2 \pi}{\lambda}\left\{(1+\cos \theta) \frac{\partial w}{\partial x}+\sin \theta \frac{\partial u}{\partial x}+|A| \frac{\partial w}{\partial x}+|B| \frac{\partial u}{\partial x}\right\} \delta x
$$




$$
A=\tan \frac{\beta}{2} \sin \theta
$$

133

$$
B=\frac{1-\cos \beta-\tan \frac{\beta}{2} \sin \theta \cos (\theta-\beta)}{\sin (\theta-\beta)}
$$

135 As in many previous cases, the initial formulation of ideas was based on the use of

136 equation 1. However, further analysis recently completed has suggested that whilst a

137 good correlation was produced between theoretical model and experimental results [13],

138 the development of the theory required further modification and optimisation to 139 improve the quality of the modelling.

140

141 The refinement introduced here has been to analyse the consequence of modelling the 142 effect of an aperture in front of the image plane. It is perhaps surprising to note that in 143 many instances, the imaging system aperture has been left out of theoretical descriptions 144 of speckle shearing theory, within many publications. This is understandable for two 145 reasons. Firstly, if an approximation is used based on the line of sight to the object 146 surface normal, and secondly, the majority of applications during the 1970's and 1980's 147 were qualitative in nature, which coincides with many of the publications in the 148 technique. Consequently, any modelling of the optical system should consider what 149 happens when imaging away from the surface normal, especially when the extremities 150 of the object are studied. 


\subsection{Modelling the influence of the image plane aperture}

153

154 The introduction of the aperture at the image plane can be seen in Figure 2. Ray 155 tracings have then been used to consider points at an arbitrary position on the planar 156 (flat) object surface, and at the extremity of the object. In order to aid clarity, the detail 157 shown at the object plane is expanded in Figure 3, specifically highlighting the 158 geometries associated with the path GOT in Figure 2.

Figures 2 and 3 show that the sensitivity vectors at the edge of illuminated area, $Q_{\mathrm{o}}$ and

$161 T_{2}$ are given by $\hat{\boldsymbol{S}}_{1}, \hat{\boldsymbol{S}}_{2}$ and $\hat{\boldsymbol{S}}_{3}$. Since the deformation is small, the values of $u$ and $w$ are 162 also small. Furthermore, $D_{1}>>D_{0}$. Therefore the angles $\angle S T_{2} Q$ and $\angle S O T$ can be 163 approximately equal and hence the distance $Q Q_{1} \approx Q Q_{2}$. The imaging angle $\varsigma^{\prime}$ is the 164 angle at point $T_{2}$ on the illuminated surface relative to the line of the optical axis and the 165 maximum value of $\varsigma^{\prime}$ is $\varsigma$, measured at the edge of illuminated object, and is given by:

$$
\varsigma=\tan ^{-1}\left[\frac{D}{2 D_{1}}\right]
$$

where $D_{1}$ is the distance from the center of the illuminated area to the center of the camera aperture and $D$ is the maximum of the inspected diameter (measured from edge to edge of the illuminated area).

The change of optical path length due to object deformation from the expanding lens $S$ to the point $Q_{1}$ and $Q_{2}$ at the image plane as shown in Figure 3 is given by:

$$
\delta l=\delta \mathbf{l} . \hat{\mathbf{n}}
$$


where $\hat{\mathbf{n}}$ is a surface unit vector. Assuming that the surface displacement vector $(\delta \mathbf{l})$ is along the surface unit vector $(\hat{\mathbf{n}})$, equation 5 can be written in form of the geometrical

178 difference of the wave propagation as:

$$
\delta l=\left(S O+O Q+Q Q_{1}\right)-\left(S T_{2}+T_{2} Q+Q Q_{2}\right)
$$

Note that at this point, the presence of the aperture modifies the modelling of the pathlength, adding additional terms when compared to the model developed without the aperture [13]. Because the path length $S G \approx S T_{2}, Q Q_{1} \approx Q Q_{2}\left(D_{1}>D_{\mathrm{o}}\right.$ and displacement $u$ is small) and $\alpha$ is small, $\delta l$ can be written as:

$$
\delta l=\left(S G+G J+J O+O T+T Q+Q Q_{1}\right)-\left(S T_{2}+T_{2} T_{1}+T_{1} Q+Q Q_{2}\right)
$$

$$
\delta l=(G J+J O+O T)-T_{2} T_{1}
$$
$(90-\beta / 2)^{\circ}$. The path length $G O$ is given by:

$$
G O=G J+J O
$$

The angle $\angle G T_{2} J=(90+\beta / 2-\theta)^{\circ}$ and using trigonometry identities:

196

$$
\frac{G J}{\sin \left(90+\frac{\beta}{2}-\theta\right)}=\frac{J T_{2}}{\sin \left(90+\frac{\beta}{2}\right)}
$$

Which gives: 


$$
G J=\frac{J T_{2}}{\sin \left(90+\frac{\beta}{2}\right)} \sin \left(90+\frac{\beta}{2}-\theta\right)
$$

$201 \quad$ Since $O O_{2}=w, O T=\frac{w}{\cos \varsigma^{\prime}}, T O_{2}=w \tan \varsigma^{\prime}$ and $T_{2} T_{1}=u \sin \varsigma^{\prime} ; J T_{2}$ can be written as:

$$
J T_{2}=w-O O_{1}
$$

The path lengths $J O$ and $O O_{1}$ are given by:

$$
J O=\frac{u-w \tan \varsigma^{\prime}}{\sin (\theta-\beta)}
$$

209

$$
O O_{1}=\frac{\left(u-w \tan \varsigma^{\prime}\right) \cos (\theta-\beta)}{\sin (\theta-\beta)}
$$

211

212 Using the relationships established in equations 9 to 13, equation 8 may be rewritten as:

$$
G O=\frac{\sin \left(90+\frac{\beta}{2}-\theta\right)}{\sin \left(90+\frac{\beta}{2}\right)}\left\{w-\left(\frac{\left(u-w \tan \varsigma^{\prime}\right) \cos (\theta-\beta)}{\sin (\theta-\beta)}\right)\right\}+\frac{u-w \tan \varsigma}{\sin (\theta-\beta)}
$$

216 
217 Therefore the change of optical pathlength $(\delta l)$ can be written as:

218

219

$$
\delta l=\frac{\sin \left(90+\frac{\beta}{2}-\theta\right)}{\sin \left(90+\frac{\beta}{2}\right)}\left\{w-\left(\frac{\left(u-w \tan \varsigma^{\prime}\right) \cos (\theta-\beta)}{\sin (\theta-\beta)}\right)\right\}+\frac{u-w \tan \varsigma}{\sin (\theta-\beta)}+\frac{w}{\cos \varsigma^{\prime}}-u \sin \varsigma^{\prime}
$$

$\delta l=\left\{\frac{\cos \frac{\beta}{2} \cos \theta+\sin \frac{\beta}{2} \sin \theta}{\cos \frac{\beta}{2}}\right\} w+\frac{w}{\cos \varsigma^{\prime}}+u\left\{\frac{1}{\sin (\theta-\beta)}-\frac{\cos \left(\frac{\beta}{2}-\theta\right)}{\cos \frac{\beta}{2}} \frac{\cos (\theta-\beta)}{\sin (\theta-\beta)}-\sin \varsigma^{\prime}\right\}$

$$
+w \tan \varsigma^{\prime}\left[\left\{\frac{\cos \left(\frac{\beta}{2}-\theta\right)}{\cos \frac{\beta}{2}} \frac{\cos (\theta-\beta)}{\sin (\theta-\beta)}\right\}-\frac{1}{\sin (\theta-\beta)}\right]
$$
be developed:

226

227

$$
\delta l=\left(\frac{1}{\cos \varsigma^{\prime}}+\cos \theta\right) w+w \tan \frac{\beta}{2} \sin \theta+w \tan \varsigma^{\prime}\left\{\frac{\cos ^{2} \theta \cos \beta}{\sin (\theta-\beta)}+\frac{[\sin \theta \cos \beta-\sin (\theta-\beta)] \sin \theta}{\sin (\theta-\beta)}\right\}
$$

228

$+w \tan \varsigma^{\prime}\left\{\frac{\tan \frac{\beta}{2} \sin \theta}{\tan (\theta-\beta)}-\frac{1}{\sin (\theta-\beta)}\right\}$

229

$$
+\left\{\frac{1}{\sin (\theta-\beta)}-\frac{\cos ^{2} \theta \cos \beta}{\sin (\theta-\beta)}-\frac{[\sin \theta \cos \beta-\sin (\theta-\beta)] \sin \theta}{\sin (\theta-\beta)}-\frac{\tan \frac{\beta}{2} \sin \theta}{\tan (\theta-\beta)}-\sin \varsigma^{\prime}\right\} u
$$

230 


$$
\left.\begin{array}{rl}
\delta l= & \left(\frac{1}{\cos \varsigma^{\prime}}+\cos \theta\right) w+w \tan \frac{\beta}{2} \sin \theta+w \tan \varsigma^{\prime}\left\{\frac{\cos \beta+\tan \frac{\beta}{2} \sin \theta \cos (\theta-\beta)-1}{\sin (\theta-\beta)}-\sin \theta\right\} \\
& +u \sin \theta+\left\{\frac{1-\cos \beta-\tan \frac{\beta}{2} \sin \theta \cos (\theta-\beta)}{\sin (\theta-\beta)}-\sin \varsigma^{\prime}\right.
\end{array}\right\}
$$

235 Consequently, equation 15 can be written as follows:

$$
\delta l=(1+\cos \theta) w+u \sin \theta+w A+u B+w A^{\prime}+u B^{\prime}
$$

where

$$
A=\tan \frac{\beta}{2} \sin \theta+\tan \varsigma^{\prime}\left\{\frac{\cos \beta+\tan \frac{\beta}{2} \sin \theta \cos (\theta-\beta)-1}{\sin (\theta-\beta)}-\sin \theta\right\}
$$

$$
A^{\prime}=\frac{1-\cos \varsigma^{\prime}}{\cos \varsigma^{\prime}}
$$

$$
B=\frac{1-\cos \beta-\tan \frac{\beta}{2} \sin \theta \cos (\theta-\beta)}{\sin (\theta-\beta)}
$$

$$
B^{\prime}=-\sin \varsigma^{\prime}
$$

244 Equation 19 represents the change of optical path length due to object deformation. It 245 can be seen that the factors $A, B, A^{\prime}$ and $B^{\prime}$ are the additional factors that are contributed 246 by the divergent non-collimated illumination wavefront and the imaging angle / aperture 247 function (varying across the illuminated surface). Compared to the original formulation of the speckle shearing interferometer theory [13], $\mathrm{A}^{\prime}$ and $\mathrm{B}^{\prime}$ are the new factors introduced as a function of the aperture at the image plane. These additional factors are related to the general OOP and IP deformations in the object deformation function that gives the influence of illumination wavefront curvature and the imaging angle to the 
252 phase change measurement. They can produce a positive or negative contributions to 253 the fringe function, depending on the direction of illumination. However, the above 254 factors are cancelled out at a point on the optical axis where the values of inclination $255 \quad$ angle $(\beta)$ and imaging angle $\left(\varsigma^{\prime}\right)$ are both equal to zero. 


\subsection{Applying lateral shear to the interferometer}

The first stage of the analysis has only considered the geometry associated with the object surface deformation and the consequent change of path length. The second stage of the analysis is to consider the correlation interferometric function of the shearing interferometer, by laterally shearing the image of the object. This is based on the assumptions that the direction of lateral shearing is in $x$-direction and the amount of shearing is $\delta x$, with the optical configuration being based on the Michelson design, as shown in Figure 1.

The speckle from a point $\mathrm{N}(x, y)$ of the first mirror of the interferometer, interferes with a speckle from a neighbouring point $\mathrm{N}^{\prime}(x+\delta x, y)$ of the second mirror. When the object is deformed, the displacement of the point $\mathrm{N}(x, y)$ will be $(u, v, w)$ and the displacement of the point $\mathrm{N}^{\prime}(x+\delta x, y)$ will be $(u+\delta u, v+\delta v, w+\delta w)$ on the image plane. The change in path length of light scattered from the mirror $\mathrm{N}^{\prime}$ is $\delta l_{2}$ and can be developed from equation 19 :

$\delta l_{2}=(1+\cos \theta)(w+\delta w)+\sin \theta(u+\delta u)+A(w+\delta w)+B(u+\delta u)+A^{\prime}(w+\delta w)+B^{\prime}(u+\delta u)$

With the assumption that the relative light path length change due to deformation is within the pixel size of the CCD camera (any issues of speckle decorrelation are therefore assumed to be minimised), the relative light path length change can be written as: 


$$
\Delta L=\delta l_{2}-\delta l
$$

283

$$
\Delta L=(1+\cos \theta) \delta w+\sin \theta \delta u+A \delta w+B \delta u+A^{\prime} \delta w+B^{\prime} \delta u
$$

285

286

287 If the optical phase change, $\Delta_{x}$, is:

288

289

$$
\Delta_{x}=\boldsymbol{k}_{x} \cdot \Delta \boldsymbol{L}
$$

290

291 where $\boldsymbol{k}_{x}$ is the wave propagation vector, $\boldsymbol{k}_{x}=2 \pi / \lambda \mathbf{n}_{x}$ and $\Delta \boldsymbol{L}$ is the displacement vector

292 (for this analysis, the wave propagation vector is assumed along the displacement 293 vector), then equation 22 can be written as:

$$
\Delta_{x}=\frac{2 \pi}{\lambda} \Delta L
$$

$$
\Delta_{x}=\frac{2 \pi}{\lambda}\left\{(1+\cos \theta) \delta w+\sin \theta \delta u+A \delta w+B \delta u+A^{\prime} \delta w+B^{\prime} \delta u\right\}
$$

$$
\frac{\Delta_{x}}{\delta x}=\frac{2 \pi}{\lambda}\left\{(1+\cos \theta) \frac{\delta w}{\delta x}+\sin \theta \frac{\delta u}{\delta x}+A \frac{\delta w}{\delta x}+B \frac{\delta u}{\delta x}+A^{\prime} \frac{\delta w}{\delta x}+B^{\prime} \frac{\delta u}{\delta x}\right\}
$$

300 where $\delta x$ is the amount of shearing in horizontal direction. If $\delta x$ is small, equation 26 can be approximated as:

$$
\frac{\Delta_{x}}{\delta x}=\frac{2 \pi}{\lambda}\left\{(1+\cos \theta) \frac{\partial w}{\partial x}+\sin \theta \frac{\partial u}{\partial x}+A \frac{\partial w}{\partial x}+B \frac{\partial u}{\partial x}+A^{\prime} \frac{\partial w}{\partial x}+B^{\prime} \frac{\partial u}{\partial x}\right\}
$$


305 which may be written in the familiar format as:

306

$$
\Delta_{x}=\frac{2 \pi}{\lambda}\left\{(1+\cos \theta) \frac{\partial w}{\partial x}+\sin \theta \frac{\partial u}{\partial x}+A \frac{\partial w}{\partial x}+B \frac{\partial u}{\partial x}+A^{\prime} \frac{\partial w}{\partial x}+B^{\prime} \frac{\partial u}{\partial x}\right\} \delta x
$$

$308 \quad$ where

$$
A=\tan \frac{\beta}{2} \sin \theta+\tan \varsigma^{\prime}\left\{\frac{\cos \beta+\tan \frac{\beta}{2} \sin \theta \cos (\theta-\beta)-1}{\sin (\theta-\beta)}-\sin \theta\right\}
$$

$$
A^{\prime}=\frac{1-\cos \varsigma^{\prime}}{\cos \varsigma^{\prime}}
$$

$$
B=\frac{1-\cos \beta-\tan \frac{\beta}{2} \sin \theta \cos (\theta-\beta)}{\sin (\theta-\beta)}
$$

$$
B^{\prime}=-\sin \varsigma^{\prime}
$$

314 The functions $A, B, A^{\prime}$ and $B^{\prime}$ in equation 28 are the OOP and IP additional sensitivity

315 factors propagated by the curvature wavefront and aperture geometrical factors, their 316 values dependant on the magnitudes of inclination angle $(\beta)$, imaging angle $\left(\varsigma^{\prime}\right)$ and the 317 illumination angle $(\theta)$. A similar relationship exists for the vertical sheared component $318 \quad(\delta y)$ 

collimated illumination

It is important at this point to test the model described in equation 28 with respect to the primary criteria of the analysis, namely differences caused by the use of collimated and non-collimated illumination. The maximum inclination angle $\beta$ depends on two variable parameters, the illuminated object diameter $(D)$ and the distance from expanding lens to the object surface $(R(L))$ :

$$
\beta=\sin ^{-1}\left[\frac{D}{2 R(L)}\right]
$$

For a given laser power the diameter of the inspected object is determined by the illuminated area on the object surface, which depends on the camera lens and the power of the expansion lens. In normal routine inspection, both of these parameters are determined based on the coverage area of the inspected object. Figure 4 represents the curvature of the illumination wavefront on the object surface is linearly dependant on the illuminated area. Equation 28 initially represents the theoretical phase function of the shearing interferometer for a non-collimated illumination wavefront, which is clearly the generalised case. There are two main components that influence the phase difference in the curvature phase function, which are contributed from the derivative out-of-plane 
344 (OOP) and the derivative in-plane (IP) components. These two components can 345 however be analyzed individually or as one function. The approach of the OOP case is 346 to analyze the functions of $A, A^{\prime}, B$ and $B^{\prime}$ in the equation 28 . This requires 347 consideration of the relationship based on the absolute values of the above parameters:

$$
\Delta_{x}=\frac{2 \pi}{\lambda}\left\{(1+\cos \theta) \frac{\partial w}{\partial x}+\sin \theta \frac{\partial u}{\partial x}+|A| \frac{\partial w}{\partial x}+|B| \frac{\partial u}{\partial x}+\left|A^{\prime}\right| \frac{\partial w}{\partial x}+\left|B^{\prime}\right| \frac{\partial u}{\partial x}\right\} \delta x
$$

Equation 30 shows that $\Delta_{x}$ is a function of $\partial w / \partial x$ and $\partial u / \partial x$, (the first partial displacement derivative components), whilst $(1+\cos \theta)$ and $\sin \theta$, are the sensitivity factors. The functions $A$ and $B$ can be treated as divergence sensitivity factors. The sensitivity factors are functions of the position of the light source, the camera and the point on the object (Figure 1).

More specifically, the values of $A$ and $B$ are the functions of object illumination angle $\theta$ and the inclination angle $\beta$ (the curvature of illumination wavefront). However the values of $A^{\prime}$ and $B^{\prime}$ are independent of the divergence illumination and only depend on the point of the illuminated surface relative to the point on the optical axis. The values of $\theta, \varsigma^{\prime}$ and $\beta$ are assumed to be independent to one another, as given by equations 4 and 29 , where $\varsigma$ and $\beta$ are independently defined. For a fixed imaging angle $\varsigma$, the maximum value of $A$ and $B$ for any value of $\theta$ and $\beta$ is unity, since the maximum value of $\beta$ is $90^{\circ}$. It is therefore important to consider equation 28 , both in terms of non-collimated and collimated illumination geometries, because in certain cases, the mathematical 
modelling of the speckle shearing interferometer will be greatly simplified, whilst in other cases, the four error term contributions $\left(A, B, A^{\prime}, B^{\prime}\right)$ will be significant. The testing of equation 28 is treated with respect to a point in three different positions; on the optical axis (object surface normal - Figure 2), at some arbitrary point away from the optical axis, and at the extremities of the object.

373

\subsection{Interferometer using non-collimated illumination}

375

376 $A^{\prime}=0$ and $B^{\prime}=0$. Therefore equation 28 reduces to the form shown in equation $\neq 0$ and $\beta \neq 0$, and equation 28 , when fully substituted, becomes:

383

384

385

$$
\Delta_{P}=\frac{2 \pi}{\lambda}\left\{(1+\cos \theta) \frac{\partial w}{\partial x}+\sin \theta \frac{\partial u}{\partial x}\right\} \delta x
$$

$$
+\frac{2 \pi}{\lambda}\left\{\left[\frac{1-\cos \varsigma^{\prime}}{\cos \varsigma^{\prime}}\right] \frac{\partial w}{\partial x}+\left[\tan \frac{\beta}{2} \sin \theta+\tan \varsigma^{\prime}\left\{\frac{\cos \beta+\tan \frac{\beta}{2} \sin \theta \cos (\theta-\beta)-1}{\sin (\theta-\beta)}-\sin \theta\right\}\right] \frac{\partial w}{\partial x}\right\} \delta x
$$




$$
\Delta_{P e}=\frac{2 \pi}{\lambda}\left\{(1+\cos \theta) \frac{\partial w}{\partial x}+\sin \theta \frac{\partial u}{\partial x}\right\} \delta x
$$


403 Again, assuming the optical phase function is at a point on the optical axis, then as in the non-collimated case, this simplifies to the form of equation 1.

406

407

The optical phase function is at a point other than on the optical axis. The imaging angle $\varsigma^{\prime} \neq 0$ and $\beta=0$, therefore the parameters $A, A^{\prime}, \mathbf{B}$ and $B^{\prime}$ will be

409 simplified to:

410

$$
A=-\tan \varsigma^{\prime} \sin \theta
$$

412

$$
A^{\prime}=\frac{1-\cos \varsigma^{\prime}}{\cos \varsigma^{\prime}}
$$

$$
B=0
$$

$$
B^{\prime}=-\sin \varsigma^{\prime}
$$

$$
\Delta_{c P}=\frac{2 \pi}{\lambda}\left\{(1+\cos \theta) \frac{\partial w}{\partial x}+\sin \theta \frac{\partial u}{\partial x}-\sin \varsigma^{\prime} \frac{\partial u}{\partial x}-\tan \varsigma^{\prime} \sin \theta \frac{\partial w}{\partial x}+\left(\frac{1-\cos \varsigma^{\prime}}{\cos \varsigma^{\prime}}\right) \frac{\partial w}{\partial x}\right\} \delta x
$$
$\neq 0$, and $\beta=0$, and equation 28 will be transformed to: 
425

$$
\Delta_{c e}=\frac{2 \pi}{\lambda}\left\{(1+\cos \theta) \frac{\partial w}{\partial x}+\sin \theta \frac{\partial u}{\partial x}-\sin \varsigma \frac{\partial u}{\partial x}-\tan \varsigma \sin \theta \frac{\partial w}{\partial x}+\left(\frac{1-\cos \varsigma}{\cos \varsigma}\right) \frac{\partial w}{\partial x}\right\} \delta x
$$

426

427

428 


\subsection{Considering the optical phase model as an error function}

429

430 From Equations 32 and 34, the relative maximum phase difference at the edge of the 431 illuminated area due to the divergent illumination wavefront can be defined, as the 432 difference of the maximum relative phase measured by divergent illumination beam, to 433 the maximum relative phase measured by collimated illumination beam:

$$
M_{r}=\frac{\Delta_{P e}-\Delta_{c e}}{\Delta_{c e}}
$$

Via substitution:

$M_{r}=\frac{\left|\tan \frac{\beta}{2} \sin \theta\right| \frac{\partial w}{\partial x}+\left|\tan \varsigma\left\{\frac{\cos \beta+\tan \frac{\beta}{2} \sin \theta \cos (\theta-\beta)-1}{\sin (\theta-\beta)}\right\} \frac{\partial w}{\partial x}\right|+\left\{\frac{1-\cos \beta-\tan \frac{\beta}{2} \sin \theta \cos (\theta-\beta)}{\sin (\theta-\beta)}\right\} \mid \frac{\partial u}{\partial x}}{(1+\cos \theta) \frac{\partial w}{\partial x}+\sin \theta \frac{\partial u}{\partial x}-\sin \varsigma \frac{\partial u}{\partial x}-\tan \varsigma \sin \theta \frac{\partial w}{\partial x}+\left\{\frac{1-\cos \varsigma}{\cos \varsigma}\right\} \frac{\partial w}{\partial x}}$

The maximum relative phase change difference value can be defined accurately if the derivative value terms $\partial w / \partial x$ and $\partial u / \partial x$ are known. However the individual derivative factors $\partial w / \partial x$ and $\partial u / \partial x$ in equation 35 are difficult to calculate simultaneously since there are typically no details of in-plane contribution of out-of-plane test object even at normal illumination angle (parallel to camera axis).

A straightforward method can be practically imposed to overcome the above difficulties, by measuring the maximum phase data using divergent illumination and the maximum phase data using collimated illumination, but maintaining all other interferometer variables as constants, as previously reported [13]. The difference of 
these two optical illumination geometries can be considered as the relative maximum phase change difference at the edge of illuminated area in the measurement analysis. divergence sensitivity factor $\left(A, B, A^{\prime}\right.$ and $\left.B^{\prime}\right)$ values from equation 32 . First consider the extreme case of the individual value of $A, B, A^{\prime}$ and $B^{\prime}$ ' when the angle between the illumination wavefront and the interferometer camera axis $\theta$ is zero and ninety degrees respectively. For the non-collimated case, with $\theta=0^{\circ}$, the factors in equation 32 become:

$$
A=-\tan \varsigma\left[\frac{\cos \beta-1}{\sin \beta}\right]=-\tan \varsigma \tan \frac{\beta}{2}
$$

$$
B=\frac{1-\cos \beta}{-\sin \beta}=-\tan \frac{\beta}{2}
$$

$$
A^{\prime}=\frac{1-\cos \varsigma}{\cos \varsigma}=\tan \frac{\varsigma}{2} \tan \varsigma
$$

And can be written as:

$$
\Delta_{x}=\frac{2 \pi \delta x}{\lambda}\left\{2 \frac{\partial w}{\partial x}+\left|\tan \frac{\beta}{2}\right| \frac{\partial u}{\partial x}+\left|\tan \varsigma \tan \frac{\beta}{2}\right| \frac{\partial w}{\partial x}+\left|\tan \frac{\varsigma}{2} \tan \varsigma\right| \frac{\partial u}{\partial x}+|\sin \varsigma| \frac{\partial u}{\partial x}\right\}
$$




$$
\Delta_{c x}=\frac{2 \pi \delta x}{\lambda}\left\{2 \frac{\partial w}{\partial x}+\left|\tan \frac{\varsigma}{2} \tan \varsigma\right| \frac{\partial u}{\partial x}+|\sin \varsigma| \frac{\partial u}{\partial x}\right\}
$$

475 If $\theta= \pm 90^{\circ}$, the factors in equation 32 can be simplified as:

476

$$
A= \pm \tan \frac{\beta}{2} \pm \tan \varsigma
$$

$$
B=\frac{1-\cos \beta-\tan \frac{\beta}{2} \sin \beta}{\cos \beta}=0
$$

$$
A^{\prime}=\frac{1-\cos \varsigma}{\cos \varsigma}=\tan \frac{\varsigma}{2} \tan \varsigma
$$

$$
B^{\prime}=-\sin \varsigma
$$

481

482 Resulting in the phase term $\Delta_{\mathrm{x}}$ being described as:

483

484

$$
\Delta_{x}=\frac{2 \pi \delta x}{\lambda}\left\{\frac{\partial w}{\partial x} \pm \frac{\partial u}{\partial x}+\left|\left(\tan \frac{\beta}{2} \mp \tan \varsigma\right)\right| \frac{\partial w}{\partial x}+\left|\tan \frac{\varsigma}{2} \tan \varsigma\right| \frac{\partial u}{\partial x}+|\sin \varsigma| \frac{\partial u}{\partial x}\right\}
$$

486 If collimated illumination is used, Equation 39 can be written as:

487

488

$$
\Delta_{c x}=\frac{2 \pi \delta x}{\lambda}\left\{\frac{\partial w}{\partial x} \pm \frac{\partial u}{\partial x}+|\tan \varsigma| \frac{\partial w}{\partial x}+\left|\tan \frac{\varsigma}{2} \tan \varsigma\right| \frac{\partial u}{\partial x}+|\sin \varsigma| \frac{\partial u}{\partial x}\right\}
$$


contributions have been modelled and are shown in Figure 5, based on an experimental configuration of a $100 \mathrm{~mm}$ illuminated diameter (planar flat object) with a source to object distance of $600 \mathrm{~mm}$ (source to image plane distance of $980 \mathrm{~mm}$ ) and an illumination angle $45^{\circ}$. Under these conditions, the relative maximum phase change contribution due to the aperture contribution using the above criteria is seen to be $0.25 \%$. this is in comparison to the $6.05 \%$ maximum relative phase change difference which is caused by the divergent aspect of the modelling for the same interferometer parameters. This part of the model was previously verified experimentally [13].

Clearly $0.25 \%$ is a small contribution to the instrument error budget. However, if the object distance decreases (object to image plane), then this element increases to $1.48 \%$ at $100 \mathrm{~mm}$. Conversely, if the object distance increases to $1000 \mathrm{~mm}$, then this error contribution reduces to $0.14 \%$. In comparison, the modelled divergent component of

505 the error term at $200 \mathrm{~mm}$ object distance is $18.4 \%$, although this has not been 506 experimentally verified, with experimentation [13] limited to 400mm object distance. 507 These results are valid for the assumption that the object surface undergoes a motion 508 dominated in the out-of-plane or surface normal direction. Further analysis is required 509 when considering objects which display predominantly in-plane motion. 
513 The growing importance of developing confidence in optical metrology data, requires a

514 better understanding of the sources of error within the instruments. Speckle shearing

515 interferometers fall into this category, because there is a significant industrial demand

516 for this type of transducer to produce quantitative data. As a prerequisite to defining

517 traceability routes to the primary standards, and even calibration artefacts, a detailed

518 error and uncertainty analysis is required for the interferometer. Some aspects of error

519 analysis have previously been completed in literature for various speckle based

520 techniques, but other elements of the speckle shearing interferometer have as yet

521 remained untouched.

An originally proposed model which described optical phase errors as a function of noncollimated object illumination for a speckle shearing interferometer, has been optimised and up-dated to include phase error terms caused by the aperture at the image plane. The development of the theoretical model has been tested by analysing different points on the object surface under collimated and non-collimated illumination conditions, 528 assuming that the surface exhibits predominantly out-of-plane deformation 529 characteristics. The modelling results have been further considered in the context of $530 \quad$ existing validating experimental data. $(0.15 \%$ at $1000 \mathrm{~mm}-1.48 \%$ at $200 \mathrm{~mm})$ to the optical phase term, which is dependant 534 on the object distance, although other contributions expressed in the model are larger.

535 Furthermore, the new terms aid the correlation between model and experimental data, 
identified in previous published work. This work is currently being extended to understand explicit issues of in-plane deformation terms and errors.

539 The new phase relationship for the speckle shearing interferometer could also be

540 regarded as the general equation for object deformation that includes all geometrical 541 parameters involved in the measurement system. However, it should be clearly 542 identified that this model is based on the analysis of flat planar surfaces or structures.

543 If objects with three dimensional relief are to be investigated, then local variations of 544 transducer sensitivity as a function of object relief, would require additional 545 compensating terms in the optical phase description.

547 Furthermore, whilst the initial elements of this model are pertinent to other wholefield 548 speckle techniques (Electronic Speckle Pattern Interferometry for instance), the final 549 elements of the model are specific to the speckle shearing interferometer dues to the 550 lateral shearing component. It should also be noted that this model has been developed 551 in isolation from other known error sources, such as the effect of higher order partial 552 derivatives, and issues such as knowing (or not knowing) the exact positions of the 553 primary optical elements of the instrument.

555 However, the value of this model is that it identifies significant error terms as a function 556 of the aperture and the divergent illumination criteria, which will contribute to the 557 whole error budget of the instrumentation. It is recognised that under certain 558 conditions, these contributions are small (large object distances), but if the parameters 559 change, then so do the error contributions. 
561 The model also provides further basis for developing error mapping routines within the 562 typical image processing software used for correlation fringe manipulation, and which 563 would compensate for specific instrumentation variables. And finally, the model helps 564 to define aspects of the full error analysis of the instrumentation, this being a 565 prerequisite for achieving the true metrological calibration-traceability of the technique.

566

567

568 


\section{Acknowledgements}

569

570 The authors would like to thank the Malaysian Government for their sponsorship of W

571 S Wan Abdullah from the Malaysian Institute for Nuclear Technology Research

572 (MINT) during the course of his research studies at Loughborough University. 


\section{References}

574

575

[1] W. Steinchen and L. Yang, 2003, Digital shearography (Bellingham, USA: SPIE

576 Press).

577

578

[2] J. S. Ibrahim, J. N. Petzing and J. R. Tyrer, 2004, Journal of Aerospace

579 Engineering, 218, 287.

580

581

582

[3] A. Martínez, R. Rodríguez-Vera, J. A. Rayas, and H. J. Puga, 2003, Optics Communications, 223, 239.

583

584

[4] R. R. Cordero, A. Martínez, R. Rodríguez-Vera, and P Roth, 2004, Optics Communications, 241, 279.

586

[5] J. M. Huntley, 1998, Journal of the Optical Society of America, 15(8), 2233.

588

589

[6] M. Owner-Petersen, 1991, Applied Optics, 30(19), 2730, 1991.

590

591

592

[7] M. Owner-Petersen, 1991, Journal of the Optical Society of America A, 8(7),

593

594

[8] T. W. Ng and F. S. Chau, 1992, Journal of Modern Optics, 39(12), 2463.

595

596

[9] Y. Iwahashi, K. Iwata, and R. Nagata, 1985, Applied Optics, 24(14), 2189.

597 
[10] N. Krishna Mohan, P. J. Masalkar, V. M. Murukeshan, and R. S. Sirohi, 1994, Optical Engineering, 33(6), 1973.

600

[11] W. Steinchen, L. X. Yang, G. Kupfer, P. Mäckel, and F. Vössing, 1998, Journal of Strain Analysis, 33(2), 171.

603

604

[12] D. Albrecht, 1999, Optics and Lasers in Engineering, 31, 63.

605

606

[13] W. S. Wan Abdullah, J. N. Petzing, and J. R. Tyrer, 2001, Journal of Modern Optics, 48(5), 757.

608

[14] W. S. Wan Abdullah, Analysis of error functions in speckle shearing interferometry, PhD Thesis, Loughborough University, UK, (2001).

611

[15] D. I. Farrant, and J. N. Petzing, 2003, Applied Optics, 42(28), 5634.

613

[16] J. Leendertz and J. N. Butters, 1973, Journal of Physics E: Scientific Instruments, 6, 1107.

616

[17] Y. Y. Hung, 1974, Optics Communications, 11(2), 132.

618

619

[18] Y. Y. Hung, and A. J. Durelli, 1979, Journal of Strain Analysis, 14(3), 81.

620

[19] Y. Y. Hung, and C. Y. Liang, 1979, Applied Optics, 18(7), 1046. 


\section{List of Figures}

623

624 Figure 1 Optical design for a Michelson based speckle shearing interferometer 625

$626 \quad$ Figure $2 \quad$ Object illumination and image plane optical considerations

627

$628 \quad$ Figure $3 \quad$ Further detail of the optical geometry

629

630 Figure 4 Relationship between the illumination diameter and the wavefront

631 inclination angle

632

633 Figure 5 Theoretical modelling of the error contribution caused by the inclusion 634 of the aperture at the image plane

635 


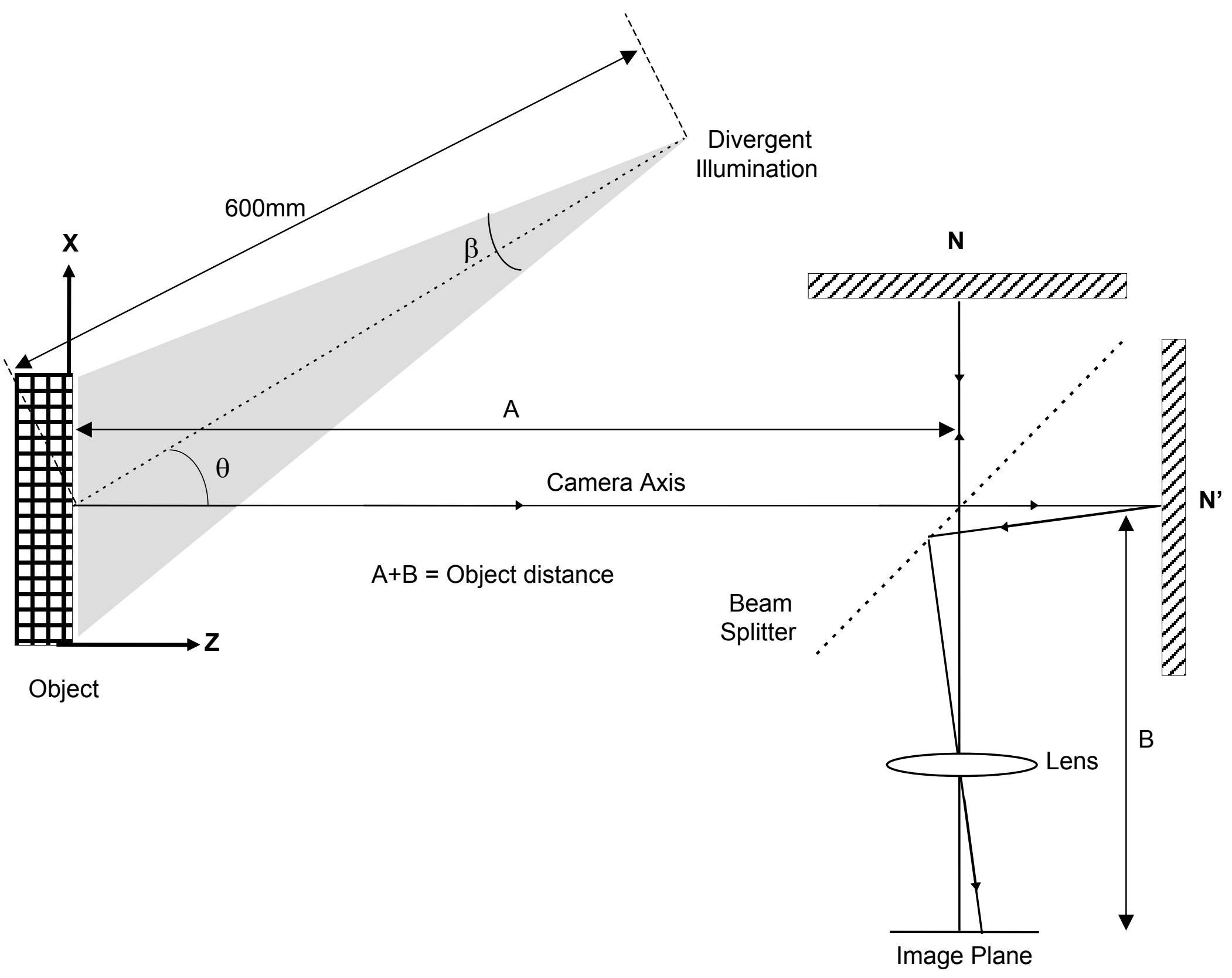




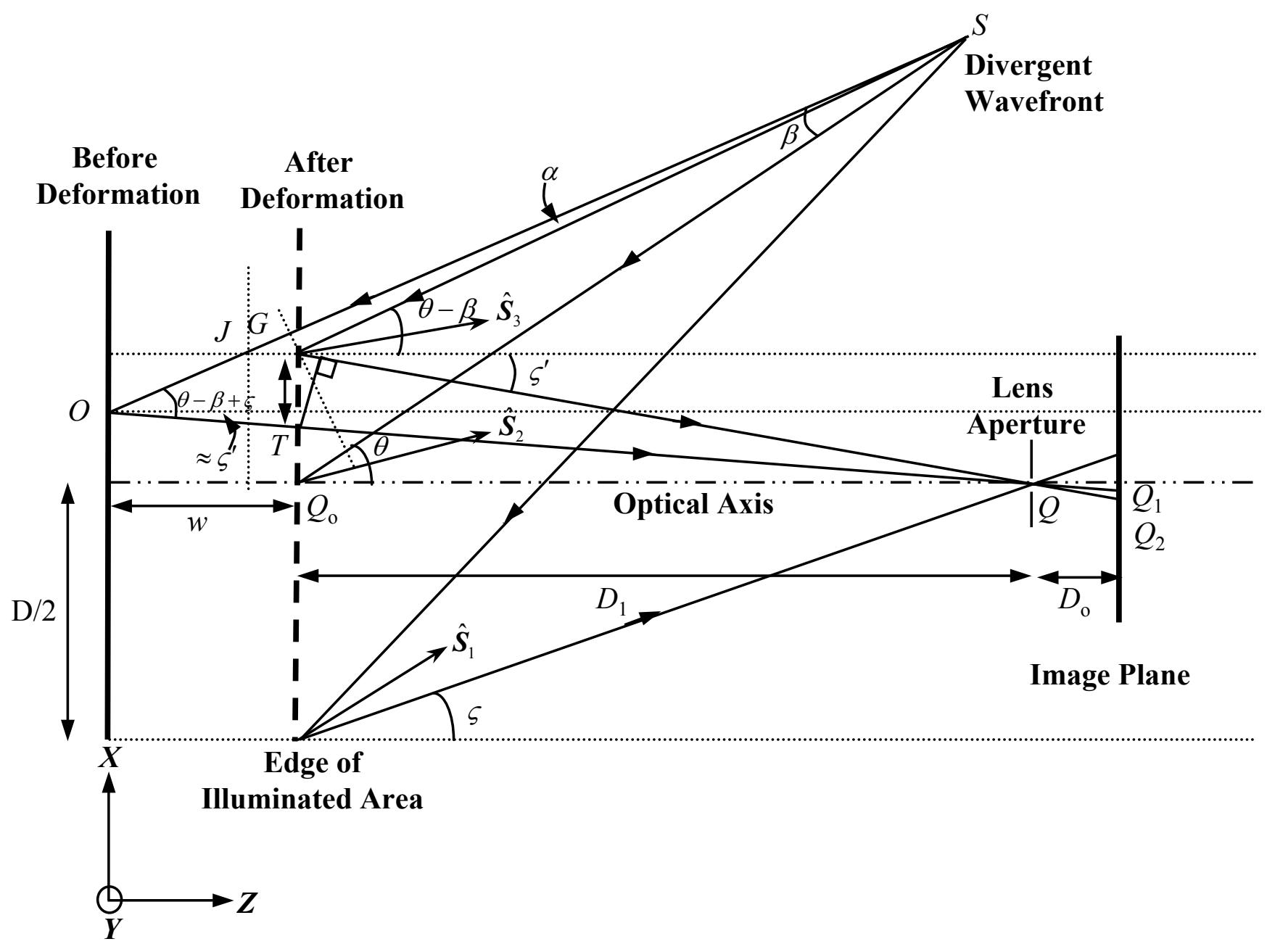



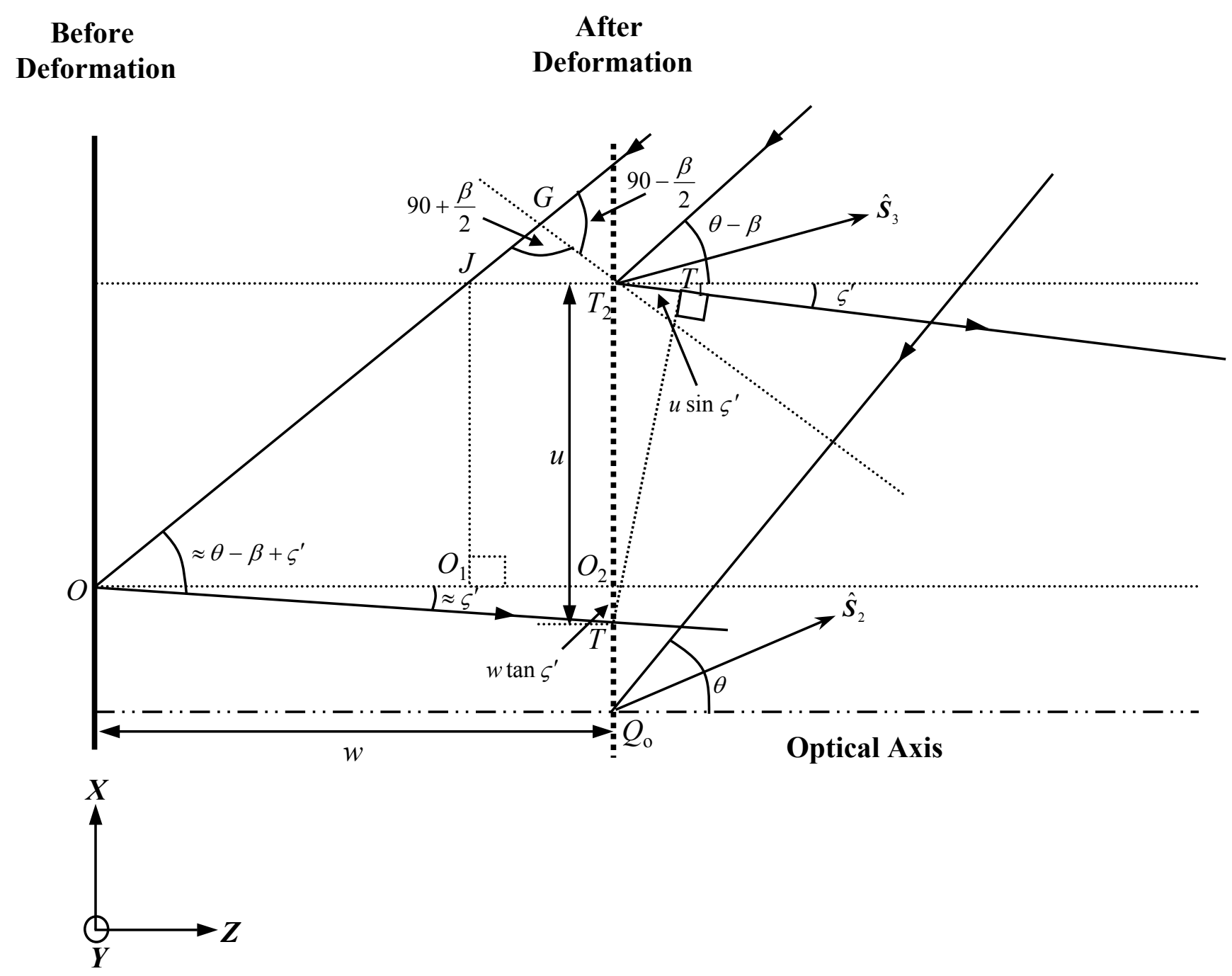


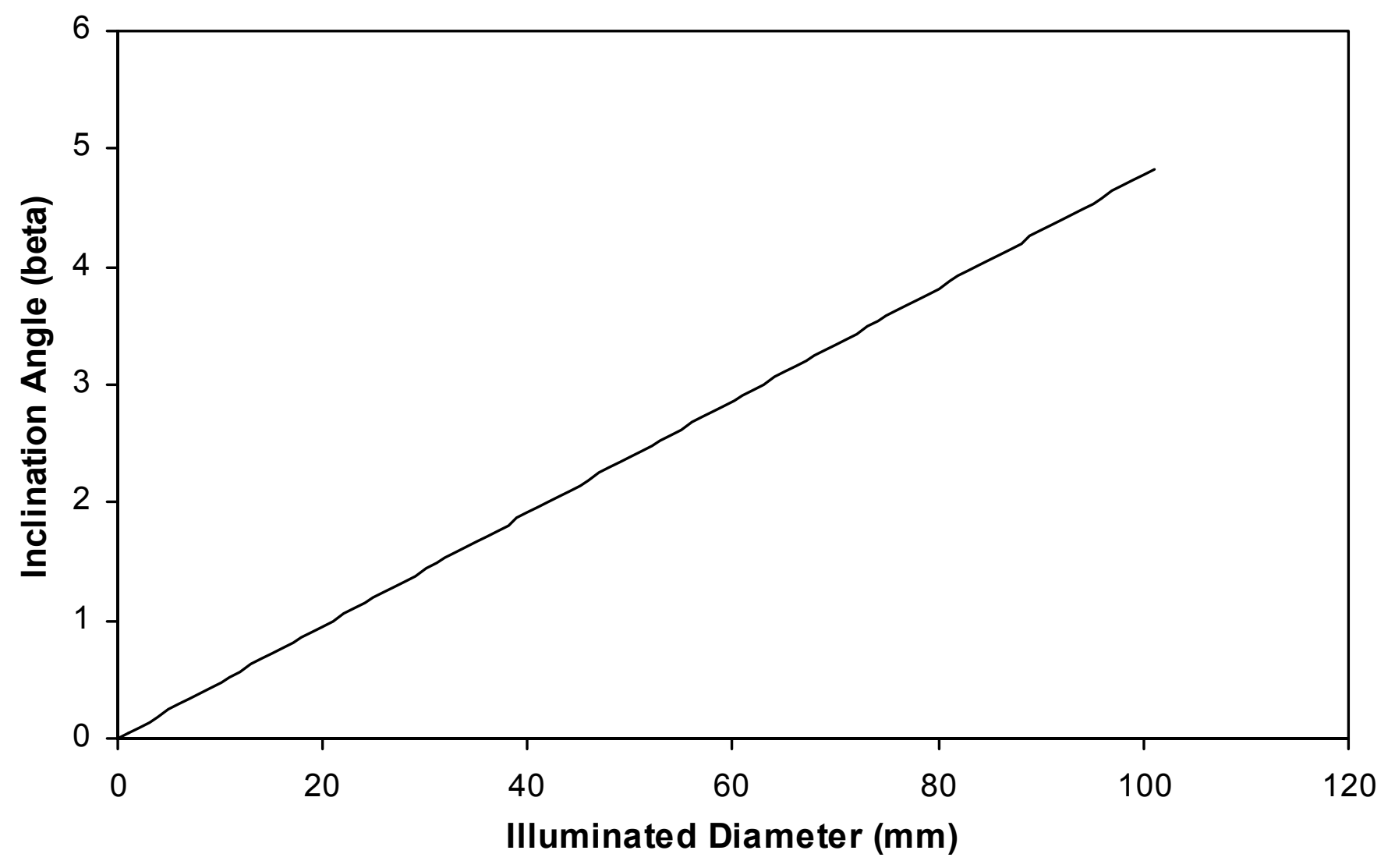




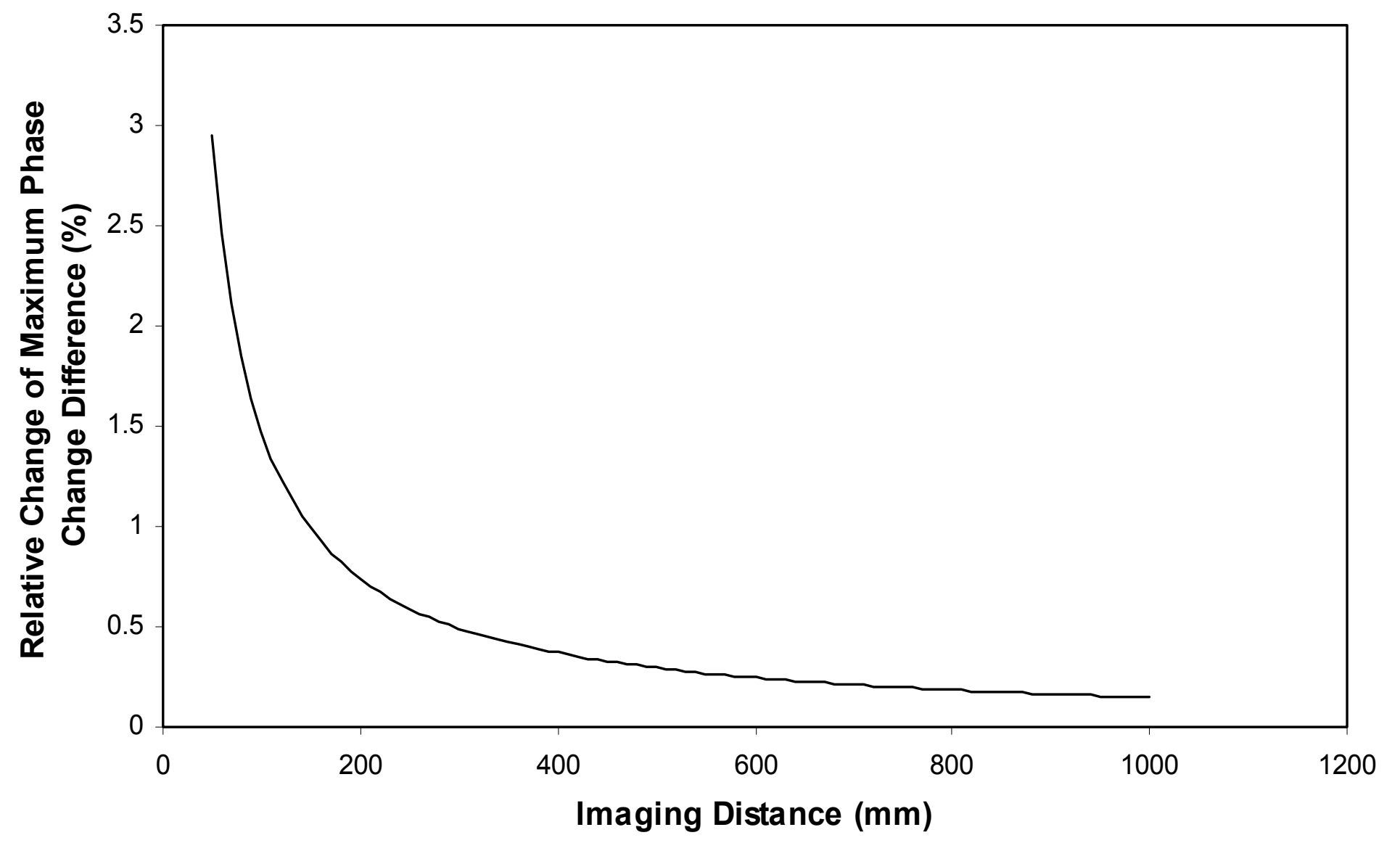

